\title{
On The Longevity Risk Assessment Under Solvency II
}

\author{
Sana Ben Salah, LaREMFiQ, University of Sousse, Tunisia
}

Lotfi Belkacem, LaREMFiQ, University of Sousse, Tunisia

\begin{abstract}
This paper deals with the longevity risk assessment within the Solvency II framework. We propose a methodology allowing obtaining longevity shocks specified by gender, age and maturity. These shocks, which are calibrated on experience mortality data relative to a French insurance company, are proved to be far away from that assumed in the standard formula and the resulting solvency capital requirement (SCR) leads to significant capital savings as compared to the standard approach.
\end{abstract}

Keywords: Solvency II; Longevity Risk; Solvency Capital Requirement

\section{INTRODUCTION}

n line with the Basel II requirements for banks, the Solvency II directive aims to review the prudential regime and ensure the financial soundness of insurance and reinsurance undertakings. Within this framework, the SCR is defined as the amount that the insurer must hold so as to remain solvent over the forthcoming 12 months and maintain its default probability below a specific threshold standing at $0,5 \%$. This regulatory capital can be determined based on either the standard approach proposed by the Committee of European Insurance and Occupational Pension Supervisors (CEIOPS), or a more sophisticated internal model. Undertakings are also allowed to use partial internal models in which some risks are internally modeled while the rest is treated in line with the standard approach. Except for the first approach, the supervisor's prior approval is usually required.

Since its approval by the Council of the European Union and the European Parliament in November 2009, the Solvency II directive has been the subject of research. In this context, Devolder (2011) casts light on the market risk and proposes to assess the insurer's solvency using various risk measures integrating the time dimension. Gatzert and Martin (2012) focus on the credit risk assessment and propose a partial internal model that allows overcoming the standard formula's weaknesses by taking into consideration all the risks inherent in a portfolio of stocks and bonds. Focusing on biometric risks, Christiansen et al. (2012) address the problem of aggregating solvency capitals using the square root formula and discuss the adequacy of the QIS (Quantitative Impact Studies) correlation matrix. Peleckienea and Peleckis (2014) present an analysis of the Solvency II Quantitative Impact Studies and discuss their effects on the international competitiveness of the European insurers.

One of the most important risks threatening life insurers is longevity risk, i.e. the risk that policy holders on average survive longer than expected. This systematic risk becomes increasingly striking with the lengthening of the human lifespan. Indeed, a part from age, time constitutes an important factor explaining the mortality evolution.

Then, as pointed out by Börger (2010) and Plat (2011), for the insurers to be shielded from mortality decreasing and to adequately assess longevity risk under the new solvency regime, a reliable stochastic mortality model must be established. In the same context, Meyricke and Sherris (2014) propose to quantify the tradeoff between the costs and benefits of using longevity-linked securities as hedging instruments.

According to the new solvency directive, longevity risk is defined as "the risk of loss, or of adverse change in the value of insurance liabilities, resulting from changes in the level, trend, or volatility of mortality rates, where a decrease in the mortality rate leads to an increase in the value of insurance liabilities" and is considered as a sub- 
module of the life underwriting risk. ${ }^{1}$ In this framework and according to the standard formula, the longevity SCR corresponds to the change in Net Asset Value (NAV) resulting from a one-off instantaneous longevity shock. ${ }^{2}$ This shock corresponds to a permanent decrease in mortality rates and is assumed to be consistent with the $0.5 \%$ value at risk of the NAV over a one year. Being set at $25 \%$ in the third quantitative impact study, the longevity stress has not been revised in the following QIS what causes numerous criticisms. In particular Börger (2010) casts light on its unrealistic structure and suggests modifying it based on the forward survival probabilities obtained with the BBRZ mortality model introduced by Bauer et al. $(2008,2010)$.

In the QIS 5, the CEIOPS proposed to review the longevity stress and consider a uniform and permanent $20 \%$ decrease in mortality rates while keeping the structure unchanged. The adequacy of this stress was discussed in Jarner and Møller (2013) who introduce a simple partial internal model for longevity risk based on the Danish longevity benchmark. The proposed longevity stress is related to the level and trend of the benchmark as well as to the experience mortality characteristics. Although simple and easy to implement, this stress depends neither on gender nor on age.

Two relevant questions arise in this context: first the uniform $20 \%$ stress is not too conservative? And second would not be more appropriate to calibrate the longevity stress at a more granular level? This paper addresses these issues and proposes assessing longevity risk over one year by generating mortality scenarios based on the Poisson log-bilinear mortality model. In this context, we propose to estimate the gender-specific longevity stress at a more granular level, allowing for different shock levels across ages and maturities. Our methodology is based on a marginal nested simulation approach consistent with the one-year value-at-risk concept of the Solvency II directive. The resulting solvency capital requirement is then compared to its counterpart obtained with the standard formula.

To the best of our knowledge, there is no published work concerned with the calibration of the age-specific longevity stress using marginal nested simulation approach based on the Poisson mortality model. Apart from discussing the adequacy of the standard longevity stress, our key contribution is to build the insurer's liabilities distribution based on stochastic mortality projections and to estimate the longevity SCR in a partial internal model context.

The models presented in this paper are fitted to the gender-specific French mortality data spanning the period 1970-2010 for the age range [0,100]. This datasets are supplied by the Human Mortality Database. ${ }^{3}$ The choice of the observation period is motivated by the fact that prior to 1970 , death rates are very instable due to the world wars. In addition to these national data, we resort to experience mortality data relative to a life annuity portfolio pertaining to a French life insurance company.

Our results give evidence that, for all ages and all maturities, the $20 \%$ standard longevity shock is more conservative than the age-specific shock coefficients resulting from our methodology. These latter are consistent with the $99,5 \%$ value at risk of the forthcoming 12 months payments distribution. In addition, we find that the partial internal model developed in this paper leads to significant capital savings as compared to the Solvency II standard formula.

These results contribute to several areas of the literature. First, we contribute to the literature on mortality modeling; we show that the Poisson mortality model is well adapted to the longevity assessment under the new solvency directive as it allows for a more realistic description of the mortality characteristics specific to elderlies. Second, our results contribute to the on-going debate on the solvency assessment; we emphasize the shortcomings of the standard approach and propose a simulation method reflecting the real longevity risk profile of the insurance company. Although prior works have investigated this issue, we extend the current literature by focusing on the longevity SCR estimation based on a marginal nested simulation approach in a partial internal model context. Our findings should be of particular interest to life insurers as well as financial institutions exposed to longevity risk.

1 CEIOPS' Advice for Level 2 Implementing Measures on Solvency II : Standard formula SCR - Article $109 \mathrm{c}$ Life underwriting risk

2 Net Asset Value : market value of the assets minus best estimate of the liabilities

$3 \mathrm{http}: / /$ www.mortality.org 
The rest of this paper is organized as follows. Section 2 deals with stochastic mortality modeling. Section 3 casts light on the adverse selection problem. Section 4 emphasizes on the longevity risk assessment under Solvency II. Section 5 reports the results and performs a comparative analysis of the SCR calculations. Finally, section 6 conducts some concluding comments.

\section{MORTALITY MODELING}

\subsection{Notations}

In the sequel of this paper, $p_{x, t}$ denotes the probability for an $x$-aged individual in year $t$ to be still alive at age $x+1$ and $q_{x, t}=1-p_{x, t}$ designates the corresponding death probability. The age specific death rate is expressed as: $m_{x, t}=\frac{D_{x, t}}{E_{x, t}}$ where $D_{x, t}$ and $E_{x, t}$ denote respectively the number of deaths and the risk exposure recorded for age $x$ during year $t$. Henceforth $\mu_{x, t}$ designates the force of mortality for age $x$ and calendar year $t$. This index measures the probability that an individual aged $x$ dies between age $x$ and $x+\Delta x$. The force of mortality is believed to be close to the age-specific death rate $m_{x, t}$ under the piecewise constant force of mortality assumption, that is: $\mu_{x+u, t+s}=\mu_{x, t}$, for $0 \leq u<1$ and $0 \leq s<1$.

\subsection{The Lee-Carter Model}

Human mortality modeling is the subject of an abundant literature and numerous stochastic models have been introduced for mortality projection purposes. The most broadly used one is that proposed by Lee and Carter (1992):

$$
\begin{aligned}
& \log \left(\mu_{x, t}\right)=a_{x}+b_{x} k_{t}+\varepsilon_{x, t} \\
& \sum_{x_{\min }}^{x_{\max }} b_{x}=1, \quad \sum_{t_{\min }}^{t_{\max }} k_{t}=0
\end{aligned}
$$

where $\mu_{x, t}$ points out the observed force of mortality for age $x$ and calendar year $t, a_{x}$ describes the average pattern of mortality by age across years, $k_{\mathrm{t}}$ reflects the variations in the mortality level over time whereas $b_{x}$ describes the deviations from the averaged pattern when $k_{\mathrm{t}}$ varies. $t_{\min }$ and $t_{\max }$ designate respectively the first and last observation years, $x_{\min }$ and $x_{\max }$ denote respectively the first and last ages taken into consideration. Finally $\varepsilon_{\mathrm{x}, \mathrm{t}}$ are error terms reflecting the specific features of an age $x$ or year $t$ not captured by the model. $\varepsilon_{\mathrm{x}, \mathrm{t}}$ are i.i.d. according to a normal distribution $N\left(0, \sigma^{2}\right)$.

Owing to its relative simplicity, this model is still considered as a reference in the longevity modeling field (see for example Lee and Carter (1992) for an application to United Kingdom's mortality data and Bravo (2010) for the Portuguese data). However, this widespread model has come under some criticism mainly related to the error homoskedasticity hypothesis assumed in the ordinary least squares estimation via singular value decomposition. In fact, death rates (in logarithm) are proved to be much more variable for elderly than for youth. To address these shortcomings, a Poisson variant of the Lee-Carter model has been proposed by Brouhns et al. (2002) while keeping the log-bilinear form unchanged. 


\subsection{The Poisson Log-Bilinear Model}

The model is described as:

$$
\begin{aligned}
& D_{x, t}: \operatorname{Poisson}\left(E_{x, t} \mu_{x, t}\right) \text { with } \mu_{x, t}=\exp \left(a_{x}+b_{x} k_{t}\right) \\
& \sum_{\min }^{x_{\max }} b_{x}=1, \quad \sum_{\text {min }}^{t_{\max }} k_{t}=0
\end{aligned}
$$

The parameters are interpreted in the same way as for the Lee-Carter model and are estimated using the maximum likelihood technique. After estimating parameters, we project mortality into the future by extrapolating the mortality trend. We resort to the Box-Jenkins methodology to model the mortality index using an adequate ARIMA model what allows, afterwards, performing simulations of the future mortality trajectory.

\subsection{Generating Prospective Life Tables}

We build $S=5000$ gender-specific prospective mortality tables for the French population based on the Poisson log-bilinear mortality model presented above. The following key steps are considered:

- $\quad$ Step 1: generating $S(S=5000)$ new matrices containing time-dependent age-specific death numbers $D_{x, t}^{(s)}$ from a Poisson distribution with parameter $\mathrm{D}_{x, t}$

- $\quad$ Step 2: estimating, for each scenario $s$, the Poisson log-bilinear model parameters

- $\quad$ Step 3: fitting an ARIMA model for each $k_{t}^{(s)}$ series considering the initial model orders

- $\quad$ Step 4: projecting $k_{t}^{(s)}$ series based on the fitted ARIMA model

- $\quad$ Step 5: finally, deducing mortality projections by computing, for each scenario, the age-specific forces of mortality, these latter are used to infer projected death probabilities as:

$\mu_{x, t}^{(s)}=\exp \left(a_{x}^{(s)}+b_{x}^{(s)} k_{t}^{(s)}\right)$

$q_{x, t}^{(s)}=1-\exp \left(-\mu_{x, t}^{(s)}\right)$

Thus, we obtain projected age-specific mortality rates for both genders what allows building projected life tables necessary for an accurate assessment of the longevity risk within the Solvency II framework. These tables are extrapolated up to age 130 using the methodology proposed by Denuit and Goderniaux (2005).

\section{THE ADVERSE SELECTION PROBLEM}

One important feature that characterizes the life insurance industry is that policyholders' mortality rates may deviate significantly from those of the overall population raising thus an adverse selection problem. The severity of this risk differs from an insurance product to another. In fact, life annuity underwriters are more likely to survive until advanced ages than the general population. Their longevity is in general higher than that expected by life tables built on the overall general population. It seems thus crucial to take this risk into consideration so as to accurately evaluate the company-specific longevity risk profile.

Nevertheless, mortality data for experience portfolios are generally not sufficient to build a vigorous experience life table. Actuaries and demographers have a tradition of using reconciliation techniques in order to relate mortality features of the group under consideration to those of a reference population. Then, it is easy to deduce experience mortality projections from those of the general (national) population:

$$
q_{x, t}^{(\exp )}=f\left(q_{x, t}^{(n a t)}\right)
$$


As the term indicates, a relational model is an expression relating a population's mortality patterns to that of another group. Ledermann and Breas (1959) made the first known contribution in this field. Various propositions have been suggested since then and numerous reviews have been written on the subject. The most famous relational model has been introduced in 1971 by Brass who suggests considering a linear relationship between the logits of the death rates of two populations. We resort here to this type of relational models to describe experience mortality features based on those of the overall French population. In this context, and within the Brass framework, the agedependent experience mortality rates are expressed as:

$$
\begin{aligned}
& \log i t\left(q_{x, t}^{\exp }\right)=\alpha+\beta \log i t\left(q_{x, t}^{\text {nat }}\right) \\
& \log i t(q)=\log \left(\frac{q}{1-q}\right)
\end{aligned}
$$

Table 1 presents the estimation results of the Brass relational model as fitted to the French general mortality data and the experience death rates.

Table 1. Brass Estimated Parameters

\begin{tabular}{lcc}
\hline & $\boldsymbol{\alpha}$ & $\boldsymbol{\beta}$ \\
\hline Males & -0.418 & 1.068 \\
Females & -0.975 & 1.091 \\
\hline Note: All parameters are significant at $1 \%$ risk level and the coefficient $\mathrm{R}^{2}$ is quite high, standing at $95,5 \%$ for men and $95,8 \%$ for \\
women
\end{tabular}
women

Based on these results, we deduce experience mortality projections from those of the general population.

\section{CALIBRATING AGE SPECIFICE LONGEVITY SHOCKS}

In the fifth quantitative impact study, the longevity shock is set to $20 \%$. This unique one-off shock is assumed to be consistent with the one-year VaR general principle of Solvency II. Aiming to carry out comparisons with the solvency II standard formula longevity shock, we propose to calibrate experience longevity stress coefficients specified by gender and age, based on projected experience death rates obtained with the Poisson mortality model and the Brass relational model.

\subsection{Methodology}

We consider for the first year an extreme longevity shock, and then deduce the next years' mortality levels conditionally to what happened in the first year. This simulation-based methodology is coherent with the general Solvency II principle and is considered as a marginal nested simulation technique where a unique risk is studied.

Within the solvency II standard formula framework, the longevity SCR is determined based on the change in net asset value resulting from a $20 \%$ reduction in mortality:

$$
S C R^{S F}=N A V-(N A V \mid \text { longevity shock })
$$

In accordance with the value at risk Solvency II general principle, this capital is assumed to reflect one-in200-year event and be an approximation of:

$$
S C R^{V a R}=\arg \min _{x}\left\{P\left(N A V_{0}-\frac{N A V_{1}}{1+i(0,1)}>x\right) \leq 0,005\right\}
$$


where $i(0,1)$ denotes the annual risk-free interest rate at time zero for maturity one year. Noting that, on the one hand, in the context of computing the longevity capital requirement under Solvency II, changes in the death rates affect only the insurer's liabilities (we consider only the biometric risk then the NAV can only be affected by a change in mortality and this effect concerns only liabilities) and on the other hand the NAV is a monotonic function of death rates, it is equivalent to consider the quantile of the NAV or that of the death rate. Thus, in the sequel of this paper, we consider a quantile of the annual death rate rather than that of the net asset value.

\subsection{Calibrating The Longevity Stress}

Having run 5000 simulations of the first year's death rate $q_{x, T+1}^{s n a t}$, we resort to the Brass Model presented and fitted above to deduce the experience mortality scenarios $q_{x, T+1}^{s} \exp$. The age-specific first year's shock coefficient is expressed as:

$$
s c_{x, T+1}^{\exp }=\left[q_{x, T+1}^{s h o c k \exp }-\frac{1}{S} \sum_{S=1}^{S} q_{x, T+1}^{\operatorname{sexp}}\right] / \frac{1}{S} \sum_{S=1}^{S} q_{x, T+1}^{\operatorname{sexp}}
$$

where $q_{x, T+1}^{\text {shock }}$ denotes the extreme first year experience scenario deduced from that relative to the overall population $q_{x, T+1}^{\text {shock } n t}$ corresponding to the $0.5 \%$ empirical quantile. $\mathrm{T}$ refers to the last available observation year $(\mathrm{T}+1$ is thus the first projection year), $\mathrm{S}$ is the total number of scenarios $(\mathrm{S}=5000), q_{0,5 \%}\left(q_{x, T+1}^{\text {nat }}\right)$ designates the $0.5 \%$ quantile of the projected death rate for age $\mathrm{x}$ and year $(\mathrm{T}+1)$ and $q_{x, T+1}^{s \text { nat }}$ is a death rate scenario. As shown in Fig.1, the first year experience shock coefficients are far from being equivalent to the standard formula longevity shock standing at $-20 \%$.

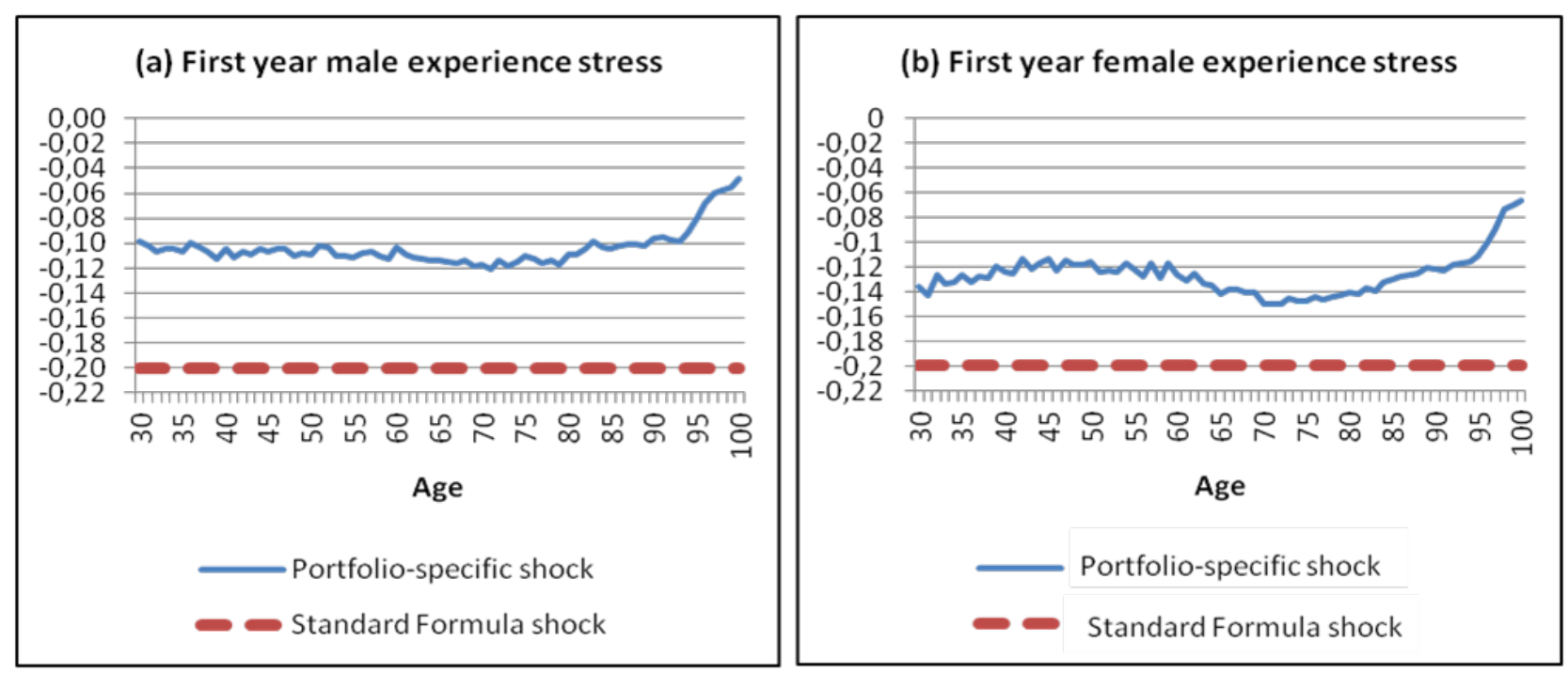

Figure 1. First Year Experience Longevity Stress 
Beyond the first projection year, we run simulations of the future death rates conditionally to the extreme first year mortality deviation. The shock coefficients are determined as:

$$
s c_{x, T+j}^{\exp }=\left[\frac{1}{S} \sum_{s=1}^{S} q_{x, T+j}^{s \text { shockexp }}-\frac{1}{S} \sum_{s=1}^{S} q_{x, T+j}^{\text {sexp }}\right] / \frac{1}{S} \sum_{s=1}^{S} q_{x, T+j}^{\operatorname{sexp}} ; j \geq 2
$$

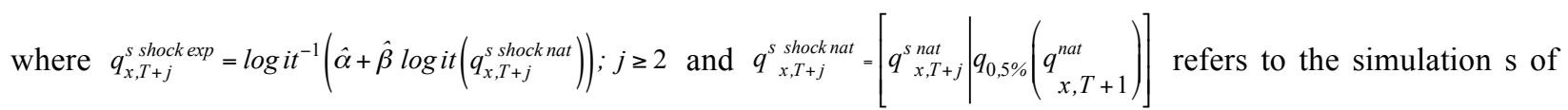
the future projected death rate determined conditionally to the stressed first year scenario. As revealed in Fig.2, the experience longevity shock coefficients differ significantly from the constant standard formula stress not only in structure but also in magnitude. In fact, these coefficients that vary outstandingly with age and maturity are considerably less restrictive than the $20 \%$ standard formula stress.

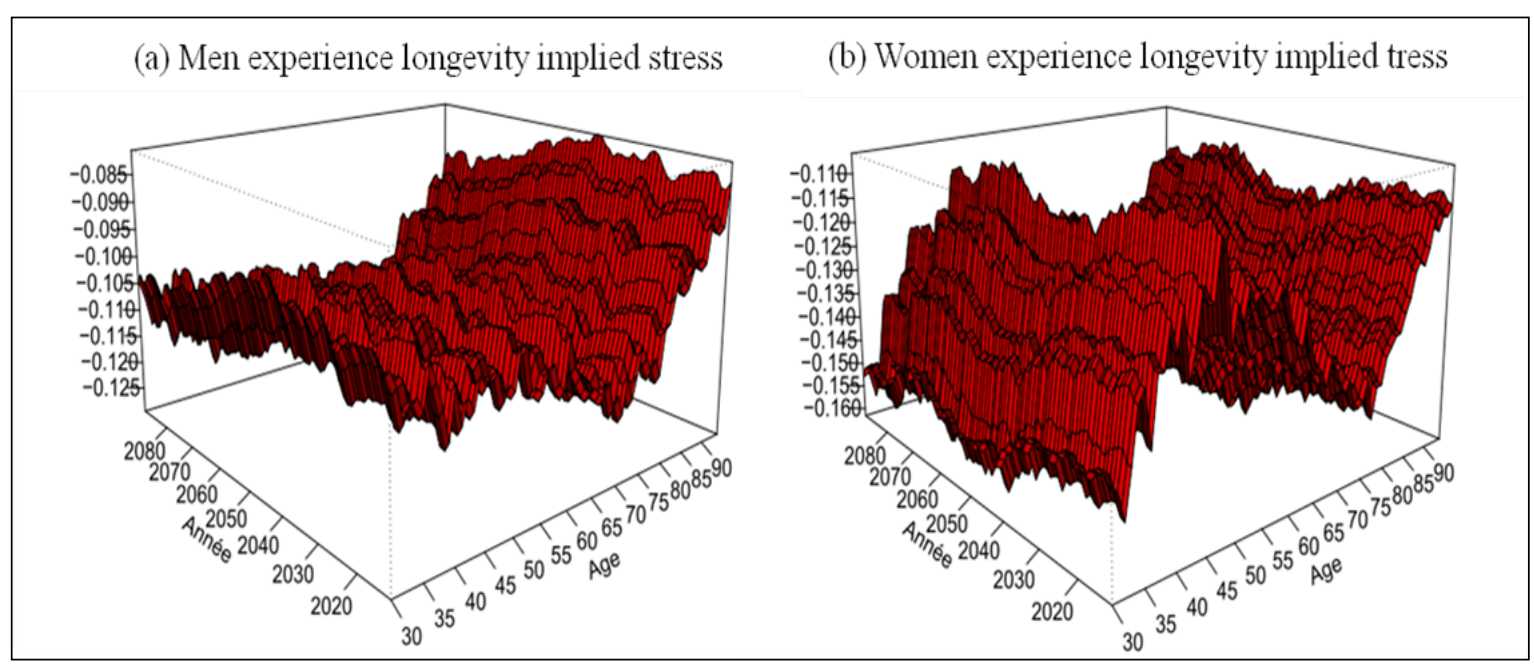

Figure 2. Experience Longevity Stress Specified By Age And Maturity For Males (A) And Females (B)

\section{SCR ESTIMATION AND COMPARATIVE ANALYSIS}

To assess the effect of using the methodology presented above on the longevity capital requirement for a life annuity portfolio, we calculate the longevity SCR based on the standard formula and the aforementioned simulation methodology. The life insurer's portfolio considered here is made of single premium in payment immediate life annuity contracts with no discretionary benefits and no options or guarantees for surrender and death benefits. These contracts pay a fixed annuity amount yearly if the insured is still alive.

Since we only consider life annuity contracts that are solely exposed to longevity risk which only affects the company's liabilities, the SCR can thus be expressed as follows:

$$
S C R^{p s}=\frac{\left(\left(B E L_{1}+C F_{1}\right) \mid \text { longevity shock }\right)}{1+i(0,1)}-B E L_{0}
$$

where $B E L_{0}$ designates the insurer's liabilities at $t=0, B E L_{1}$ denotes the company's best estimate liabilities at $t=1$ and $C F_{l}$ designates the first projection year best estimate payment. ${ }^{4} i(t, \mathrm{~T})$ is the annual interest rate for maturity $\mathrm{T}$ at time $t(t \leq \mathrm{T})$ deduced from the QIS5 term structure for EU with $100 \%$ allowance for illiquidity premium. The

${ }^{4} \mathrm{CF}_{1}>0$, the life insurance contracts into consideration are in payment and we do not consider new business. 
quantity $\left(\left(B E L_{1}+C F_{1}\right) \mid\right.$ longevity shock $)$ is calculated based on the experience stressed death rates. This quantity is consistent with the $99,5 \%$ quantile of the insurer's liability distribution over one year. For an immediate life annuity contract paying a fixed amount $\boldsymbol{a}$ yearly, the best estimate liabilities at time $t$ are:

$$
B E L_{t}=\sum_{T>t} a \cdot \frac{1}{(1+i(t, T))^{T-t}} \cdot E\left[{ }_{T} p_{x, t} \mid F_{t}\right]
$$

where $F_{t}$ expresses the information available at time $t$ and ${ }_{T} p_{x, t}$ is the probability for an $x$-aged insured person at $t$ to be still alive at the age $x+T$ :

$$
{ }_{T} p_{x, t}=\prod_{s=0}^{T-1} p_{x+s, t+s}
$$

$p_{x, t}$ denotes the one-year survival probabilities obtained from experience mortality projections. From the central and stressed experience death rates for both genders, we deduce 5000 scenarios of the survival probabilities what allows obtaining a distribution of the insurer's liabilities over one year. Table 2 reports the insurer's best estimate liabilities at time zero as well as the SCR obtained based on the liabilities distribution over one year $\left(\mathrm{SCR}^{\mathrm{ps}}\right)$ and on the standard approach $\left(\mathrm{SCR}^{\mathrm{SF}}\right)$. We can observe that $\mathrm{SCR}^{\mathrm{ps}}$ is about $44 \%$ lower than its counterpart based on the standard formula indicating that the $20 \%$ shock overestimates the portfolio's longevity risk as compared to the oneyear VaR-based approach. This deviation may be caused, in part, by the structure of the data as well as the assumptions surrounding mortality projections. In fact, the standard formula shock was calibrated on the unisex mortality data for nine populations (DE, FR, England \& Wales, ES, IT, SE, PL, HU, CZ) spanning the period 19922006. In addition, annual mortality improvements for the aforementioned countries are assumed to follow a Normal

\begin{tabular}{|c|c|c|c|c|}
\hline$(€)$ & BELO & $\mathrm{SCR}^{\mathrm{PS}}$ & $\mathbf{S C R}^{\mathrm{SF}}$ & $\left(\mathrm{SCR}^{\mathrm{PS}}-\mathrm{SCR}^{\mathrm{SF}}\right) / \mathrm{SCR}^{\mathrm{SF}}$ \\
\hline Males & 1020674 & 53996 & 102499 & $-47.3 \%$ \\
\hline Females & 5370109 & 255985 & 452698 & $-43.4 \%$ \\
\hline Total & 6390783 & 309981 & 555197 & $-44.1 \%$ \\
\hline \multicolumn{5}{|c|}{$\begin{array}{l}\text { The table presents the portfolio's best estimate liabilities along with the longevity SCRs resulting from the application of the } \\
\text { standard for } \\
\text { mula }\left(\mathrm{SCR}^{\mathrm{SF}}\right) \text { and the simulation methodology proposed in this paper }\left(\mathrm{SCR}^{\mathrm{PS}}\right) \text {. The relative spread between these capitals is } \\
\text { reported in the last column. }\end{array}$} \\
\hline
\end{tabular}
distribution.

\section{CONCLUSION}

This paper investigates the effects of using a partial internal model in the SCR estimation under Solvency II. In this context, and according to the standard formula, the longevity SCR is determined as the deviation in the net asset value due to a $20 \%$ permanent reduction in death rates for all ages. Although simple and easy to implement, this scenario-based approach comes under some criticism related mainly to the structure and magnitude of the longevity stress.

Given that the goal is for the SCR to reflect the one-year $99,5 \%$ percentile of the forthcoming 12 months payments distribution, we have proposed a methodology allowing obtaining experience longevity shocks specified by gender, age and maturity. Based on the Poisson log-bilinear mortality model, we have generated 5000 genderspecific prospective mortality life tables for the overall French population via stochastic projection techniques. Afterwards, we resorted to a Brass-type relational model to perform experience mortality projections based on the French overall population mortality features what allowed avoiding the anti selection problem. The insurer's liabilities distribution over one year has been obtained based on a marginal nested simulation technique consistent with the one-year value at risk Solvency II general principle. After computing the extreme first year mortality scenario, we have ran simulations for the subsequent periods conditional to what happened in the first year. Then, longevity shocks have been deduced from central and stressed death rates. These coefficients, specified by age, 
maturity and gender, are considerably less conservative than the standard formula stress standing at $-20 \%$ for all ages and maturities. This methodology is proved to produce significant capital savings for the longevity risk as compared to the standard formula approach.

Overall, the findings of this paper give evidence that the standard formula is unable to reflect the insurer's real risk profile and highlight the extent to which this approach overestimates the solvency capital requirement. The partial internal model proposed in this paper allows for a more realistic description of the insurer's situation over one year and thus a more accurate estimation of the longevity SCR. Our results are relevant to the problem of assessing the insurer's ruin probability over one year as they are obtained based on a sophisticated methodology particularly adapted to the longevity risk evaluation under Solvency II.

\section{AUTHOR INFORMATION}

Sana Ben Salah is a researcher, member of Research Laboratory of Economy, Management and Quantitative Finance (LaREMFiQ), University of SOUSSE-Tunisia. His research interests include risk management in finance and insurance, life insurance, non life insurance, quantitative methods, statistics, biometric risks, mortality modeling, mortality-linked securities, financial instruments and capital markets. E-mail: $\underline{\text { sana.b.salah@gmail.com }}$

Lotfi Belkacem is a full professor of Quantitative Methods at the Institute of High Commercial Studies (IHEC), University of Sousse. Currently, $\mathrm{He}$ is the Director of Reseacrh Laboratory of Economy, Management and Quantitative Finance (LaREMFiQ). He is also a member and expert of the National Council of Statistics. His research focuses on issues related to statistics, entrepreneurship, finance, insurance, education and employment economics. He is also author of several scientific publications in indexed international reviews. He is national and international expert in statistical surveys, entrepreneurship, education, job training, employment qualifications, risk management and evaluation of higher education. E-mail: lotfi.belkacem@yahoo.fr

\section{REFERENCES}

Bauer, D., Börger, M., Ruß, J., Zwiesler, H.J. (2008). The volatility of mortality. Asia-Pacific Journal of Risk and Insurance, 3 (1) 172-199.

Bauer, D., Börger, M., Ruß J. (2010). On the Pricing of longevity-linked securities. Insurance: Mathematics and Economics, 46 (1) 139-149.

Börger, M. (2010). Deterministic shock vs. stochastic value-at-risk-an analysis of the Solvency II standard model approach to longevity risk. Blätter der DGVFM (Springer), 31(2) 225-259.

Bravo, J., M. (2010). Lee-Carter mortality projection with "Limit Life Table". Joint Eurostat/UNECE Work Session on Demographic Projections, April 28-30, Lisbon, Portugal.

Brouhns, N., Denuit, M., Vermunt, J.K. (2002). A Poisson log-bilinear regression approach to the construction of projected life tables. Insurance: Mathematics and Economics, 31(3) 373-393.

CEIOPS, QIS 5 - technical specifications. 2010.

Christiansen, M.C., Denuit, M.M., Lazar, D. (2012). The Solvency II square-root formula for systematic biometric risk. Insurance: Mathematics and Economics, 50(2) 257-265.

Denuit, M., Goderniaux, A.C. (2005). Closing and projecting lifetables using log-linear models. Bulletin of the Swiss Association of Actuaries, 1, 29-49.

Devolder, P. (2011). Revised version of: Solvency requirement for long term guarantee: risk measure versus probability of ruin. European Actuarial Journal, 1(2) 200-214.

Gatzert, N., Martin, M. (2012). Quantifying credit and market risk under Solvency II: Standard approach versus internal model. Insurance: Mathematics and Economics, 51(3) 649-666.

Jarner, S., Møller, T. (2013). A partial internal model for longevity risk. Scandinavian Actuarial Journal, 1-31.

Ledermann, S., Breas, J. (1959). Les dimensions de la mortalité. Population 4, 637-682.

Lee, R.D., Carter, L.R. (1992). Modelling and forecasting U.S. mortality. Journal of the American Statistical Association 87(419) 659-675.

Meyricke, R., Sherris, M. (2014). Longevity risk, cost of capital and hedging for life insurers under Solvency II. Insurance: Mathematics and Economics, 55, 147-155.

Peleckienėa, V., Peleckis, K. (2014). Solvency II assumptions for increasing the international competitiveness of EU insurance industry. Procedia-Social and Behavioral Sciences, 110, 822-831. 


\section{NOTES}

